\title{
A tough graphene nanosheet/hydroxyapatite composite with improved in vitro biocompatibility
}

\author{
Lv Zhang ${ }^{a}$, Weiwei Liu ${ }^{a}$, Chunguang Yue ${ }^{a}$, Taihua Zhang ${ }^{b}$, Pei Li ${ }^{a}$, Zhanwen Xing ${ }^{a}$, \\ Yao Chen ${ }^{a, *}$ \\ a BioManufacturing Research Centre, School of Mechanical and Electric Engineering, Soochow University, 178 East Ganjiang Road, \\ Suzhou 215021, China \\ $\mathrm{b}$ The State Key Laboratory of Nonlinear Mechanics, Institute of Mechanics, Chinese Academy of Sciences, Beijing 100191, China
}

\section{A R T I C L E I N F O}

Article history:

Received 1 January 2013

Accepted 25 April 2013

Available online 1 May 2013

\begin{abstract}
A B S T R A C T
Mechanical properties and in vitro biocompatibility of graphene/hydroxyapatite (HA) composite synthesized using spark plasma sintering (SPS) are reported in this study. Raman spectroscopy corroborated that graphene nanosheets (GNSs) survived the harsh processing conditions of the selected SPS processing parameters. A $1.0 \mathrm{wt} . \%$ GNS/HA composite exhibits $\sim 80 \%$ improvement in fracture toughness as compared to pure HA. GNS pull-out, grain bridging by GNS, crack bridging and crack deflection are the major toughening mechanisms that resist crack propagation. In vitro osteoblast growth tests illustrate that the added GNSs contribute to the improvement of both osteoblast adhesion and apatite mineralization. Therefore, the GNS/HA composite is expected to be a promising material for load-bearing orthopedic implants.
\end{abstract}

(C) 2013 Elsevier Ltd. All rights reserved.

\section{Introduction}

Graphene, a monolayer of $\mathrm{sp}^{2}$-hybridized carbon atoms arranged in a two-dimensional lattice, has drawn much attention in the composite field as reinforcement for structural composites due to its combination of excellent mechanical properties (e.g., tensile strength $130 \mathrm{GPa}$ and Young's modulus 0.5-1 TPa) and very high specific surface area (up to $2630 \mathrm{~m}^{2} \mathrm{~g}^{-1}$ ) [1-3]. In particular, the high specific surface area of graphene, inherent to its two-dimensional lattice geometry, imparts strong interfacial bonding with the matrix phase and effective load transfer from the matrix to graphene [4]. Graphene nanosheets (GNSs) with a thickness of approximately 1-10 nm, also called as graphene nanoplatelets (GNPs) or graphene platelets (GPLs), are generally composed of a few graphene layers and display compatible properties similar to that of monolayer graphene. Furthermore, it is worth to note that GNSs are much easier to produce and handle. Very re- cently, GNSs have been widely employed as nanofillers to polymers [5,6], metals $[7,8]$ and ceramics [9-11] to produce composites with tailored mechanical properties.

Due to its chemical composition (Ca/P ratio of 1.67$)$ and crystal structure that are similar to the apatite in human skeletal system, hydroxyapatite (HA), with excellent bioactivity and osteoconductivity, is suitable for osteoblast adhesion and proliferation, new bone growth and integration [12], and therefore is recognized as one of the most promising orthopedic biomaterials. However, the intrinsic brittleness of HA, i.e., low fracture toughness and low toughness-induced poor wear resistance, still restricts its clinical applications. Therefore, toughening of HA with a second phase such as alumina, yttria stabilized zirconia, titania and carbon nanotubes (CNTs) has been extensively explored to overcome the deficiencies of pure HA [13].

Among HA based composites, much recent attention has been devoted to the CNT/HA composites. Previous

* Corresponding author: Fax: +86512 67587216.

E-mail address: chenyao@suda.edu.cn (Y. Chen).

0008-6223/\$ - see front matter (c) 2013 Elsevier Ltd. All rights reserved.

http://dx.doi.org/10.1016/j.carbon.2013.04.074 
investigations have demonstrated that CNT/HA composite possesses an improved fracture toughness compared to monolithic HA [13]. This is due to the significant role of CNTs in improving fracture toughness of the composite based on basic toughening mechanisms such as CNT pull-out, crack bridging and crack deflection [13]. CNT/HA composites also display an improved osteoblast proliferation and differentiation in vitro $[14,15]$. However, the biocompatibility of CNTs is still under debate due to their cytotoxic responses in organic environment, though some researchers have ascribed the cytotoxicity of CNTs to the presence of metallic catalyst particles rather than CNT itself [13]. Therefore, an ideal reinforcement material for HA should not only significantly improve the mechanical properties, but also can retain the original biocompatibility of HA [13].

Recently, Rafiee et al. [5] have illustrated that crack deflection is more effective for sheet-like reinforcement than for tubular-like reinforcement, strongly implying that GNSs exhibit more significant toughening effect on brittle materials than do CNTs. On the other hand, graphene can be synthesized in relatively pure ways such as growth by chemical vapor deposition, micromechanical exfoliation of graphite and growth on crystalline silicon carbide [16]. Hence, it is expected that graphene shows little cytotoxicity due to few metallic catalyst particles associated with its production. Recent investigations have revealed that graphene and graphene based composites possess a series of merits, e.g., no toxic for human osteoblasts [17], excellent antibacterial property [18], suitable for adhesion and proliferation of osteoblasts [18], and the ability of apatite mineralization [19]. To the best of our knowledge, few investigations have been made into a free-standing GNS/HA composite.

Spark plasma sintering (SPS) is a novel method that can simultaneously employ pressure and electrical current, and can complete the consolidation in few minutes at a relative low temperature. Due to low sintering temperature and short sintering time, SPS can inhibit the grain growth and subsequently improves the mechanical properties. Recent investigations indicate that the main SPS processing parameters for consolidation of HA and HA composites are sintering temperature of $900-1250{ }^{\circ} \mathrm{C}$ and applied pressure of $7.5-120 \mathrm{MPa}$ [13]. For a GNS/HA composite, it is worth to pointed out that SPS processing parameters should be further optimized to avoid the damage of the specific structure of GNSs.

In view of the present scenario, the aim of this research is to explore the potential of using GNSs as reinforcement to HA for load-bearing orthopedic applications. HA and GNS/HA composites are fabricated using SPS. The mechanical properties and in vitro biocompatibility of the sintered samples are evaluated. The role of the GNSs and their contributions towards the mechanical response and in vitro biocompatibility of the HA composite is also analyzed.

\section{Experimental procedures}

\subsection{Material preparation}

HA nanorods (length: $\sim 100 \mathrm{~nm}$, diameter: $\sim 30 \mathrm{~nm}$ ) from Nanjing Emperor Nano Material (Nanjing, China) and GNSs (thickness: $\sim 0.8 \mathrm{~nm}$, diameter: $0.5-2 \mu \mathrm{m}$ ) from ACS Material (MA, USA) were employed as precursor materials. Fig. 1a shows scanning electron microscopy (SEM; Hitachi S-4700, Japan) image of as-received GNSs.

GNS agglomerates were firstly ultrosonicated for $30 \mathrm{~min}$ in water with a concentration of about $0.1 \mathrm{mg} / \mathrm{ml}$, in which sodium dodecyl-benzene sulfonate (SDBS) was used as dispersant. Then, HA nanopowders were added into the GNS suspension followed by another $1 \mathrm{~h}$ ultrosonication. The composite powder was allowed to settle and the excess solvent was drained. Finally, the powder was dried in an oven at $120^{\circ} \mathrm{C}$. The compositions chosen here were pure HA, 0.5 wt. $\%$ GNS/HA and 1.0 wt.\% GNS/HA.

\subsection{Spark plasma sintering processing}

The resulting powders were loaded into a graphite die $(20 \mathrm{~mm}$ inner diameter), in which graphite papers were placed between the powders and die/punches for easy specimen removal. The powders were consolidated in an SPS apparatus (Dr. Sinter 1050, Sumitomo Coal Co., Ltd., Japan) using a maximum temperature of $1150{ }^{\circ} \mathrm{C}$ and a holding pressure of $40 \mathrm{MPa}$. A heating rate of $150^{\circ} \mathrm{C} / \mathrm{min}$ was employed until $1050^{\circ} \mathrm{C}$ was reached, with $1150^{\circ} \mathrm{C}$ being attained in the next minute; this maximum temperature was then kept for $3 \mathrm{~min}$. The samples were then furnace cooled to ambient temperature.

\subsection{Structural characterizations}

Sintered samples were polished to remove the graphitic contamination on their surfaces. Phase constituents of the sintered samples were identified by X-ray diffraction (XRD; X'Pert-Pro MPD, The Netherlands) using $\mathrm{Cu} \mathrm{K} \alpha$ radiation with a scanning rate of $2 \% \mathrm{~min}$ at a range from $20^{\circ}$ to $70^{\circ}$. MicroRaman spectroscopy (JR HR800, France) was employed to confirm the existence of GNSs using an Argon ion laser of wavelength $514.5 \mathrm{~nm}$ and an acquisition time of $10 \mathrm{~s}$. Fractographic observations of sintered samples and the propagation paths of crack induced by Vickers indentation were analyzed using SEM.

\subsection{Mechanical measurements}

Nanoindentation experiments were conducted using $\mathrm{NHT}^{2}$ nanoindentation tester (CSM, Switzerland) with a Berkovich tip at a maximum load of $10 \mathrm{mN}$ (ramp load of $1 \mathrm{mN} / \mathrm{s}$ and a dwell time of $15 \mathrm{~s}$ ). Elastic modulus was calculated from the load-displacement curve using Olive-Pharr method. The fracture toughness was then calculated using following equation:

$K_{\text {IC }}=0.016\left(\frac{E}{H}\right)^{0.5}\left(\frac{P}{c^{1.5}}\right)$

where $H$ is the microhardness, $E$ is the elastic modulus, $P$ is the applied load and $2 c$ is the total length of indentationinduced crack $(2 c=2 l+2 a)$, where $l$ is the length of the crack from the indentation corner, and $2 a$ is the indentation diagonal). Microhardness was measured using a microhardness tester (HXD-1000 TMC, Shanghai Taiming Optical Instrument 

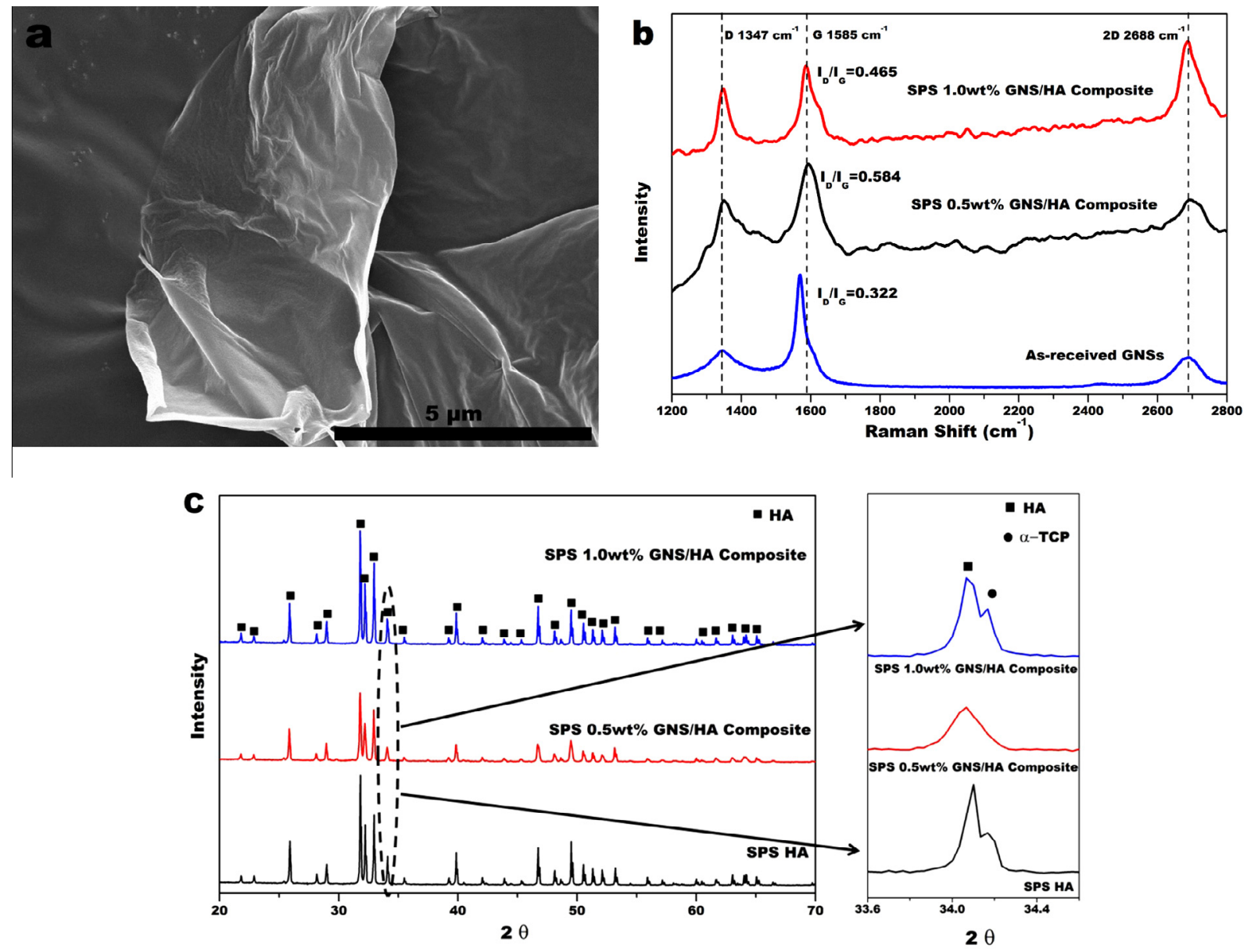

Fig. 1 - (a) SEM image of starting GNS powders, (b) Raman spectra of as-received GNSs, 0.5 wt.\% GNS/HA and 1.0 wt.\% GNS/HA composites, and (c) XRD results of sintered HA, 0.5 wt.\% GNS/HA and 1.0 wt.\% GNS/HA composites.

Co., Ltd., China) with a Vickers probe and the application of a $3 \mathrm{~N}$ load applied for a dwell time of $15 \mathrm{~s}$.

\subsection{Cell culture}

Mouse osteoblast cells (MC3T3-E1) were cultured in alpha minimum essential medium (Thermo Scientific, USA) supplemented with $10 \%$ fetal bovine serum (Invitrogen, USA). The cells were incubated at $37 \pm 1^{\circ} \mathrm{C}$ in a humidified atmosphere of $5 \% \mathrm{CO}_{2}$. When the cultured cells reached confluence, they were detached using $0.25 \%$ trypsin-ethylenediaminetetraacetic acid (Invitrogen, USA) solution to provide adequate numbers of cells for the following studies.

\subsection{Cell adhesion}

Before cell adhesion, the sintered samples were grounded to eliminate the difference in surface roughness. Samples were seeded into a 24-well plate using a cell concentration of $1 \times 10^{5}$ cells $\mathrm{cm}^{-2}$, and were allowed to attach at $37 \pm 1{ }^{\circ} \mathrm{C}$ in a humidified atmosphere of $5 \% \mathrm{CO}_{2}$ for $6 \mathrm{~h}$. Samples were then rinsed several times with phosphate-buffered saline to remove unattached cells and then fixed in ice-cold $4 \%$ paraformaldehyde for $20 \mathrm{~min}$. Fluorescence microscope (Olympus IX71, Japan) and SEM were used to investigate cell adhesion.

\subsection{Apatite mineralization}

The osteoconductivity of the as-received GNSs and as-sintered samples were assessed by apatite mineralization in a simulated body fluid (SBF) solution, which was prepared according to the well-known Kokubo composition [20]. As-received GNSs and as-sintered samples were soaked in SBF and kept at $37 \pm 1{ }^{\circ} \mathrm{C}$ in a humidified atmosphere of $5 \% \mathrm{CO}_{2}$ for 7 days. After the soaking period, samples were carefully washed with deionized water three times and dried at ambient temperature. Apatite mineralization and its morphology were carefully examined using SEM and transmission electron microscopy (TEM; JEM-2010, Japan) with energy dispersive X-ray energy spectroscopy (EDS) attachment (OXFORD Instrument, INCA, UK).

\section{Results and discussion}

\subsection{Microstructural characteristics and mechanical properties}

Raman measurement was performed to confirm the existence of GNSs. Fig. $1 b$ depicts the presence of D, G and 2D peaks, in which $\mathrm{G}$ peak is from the in-plane $\mathrm{C}-\mathrm{C}$ bond stretching in graphene, D peak is related to the defects in the 

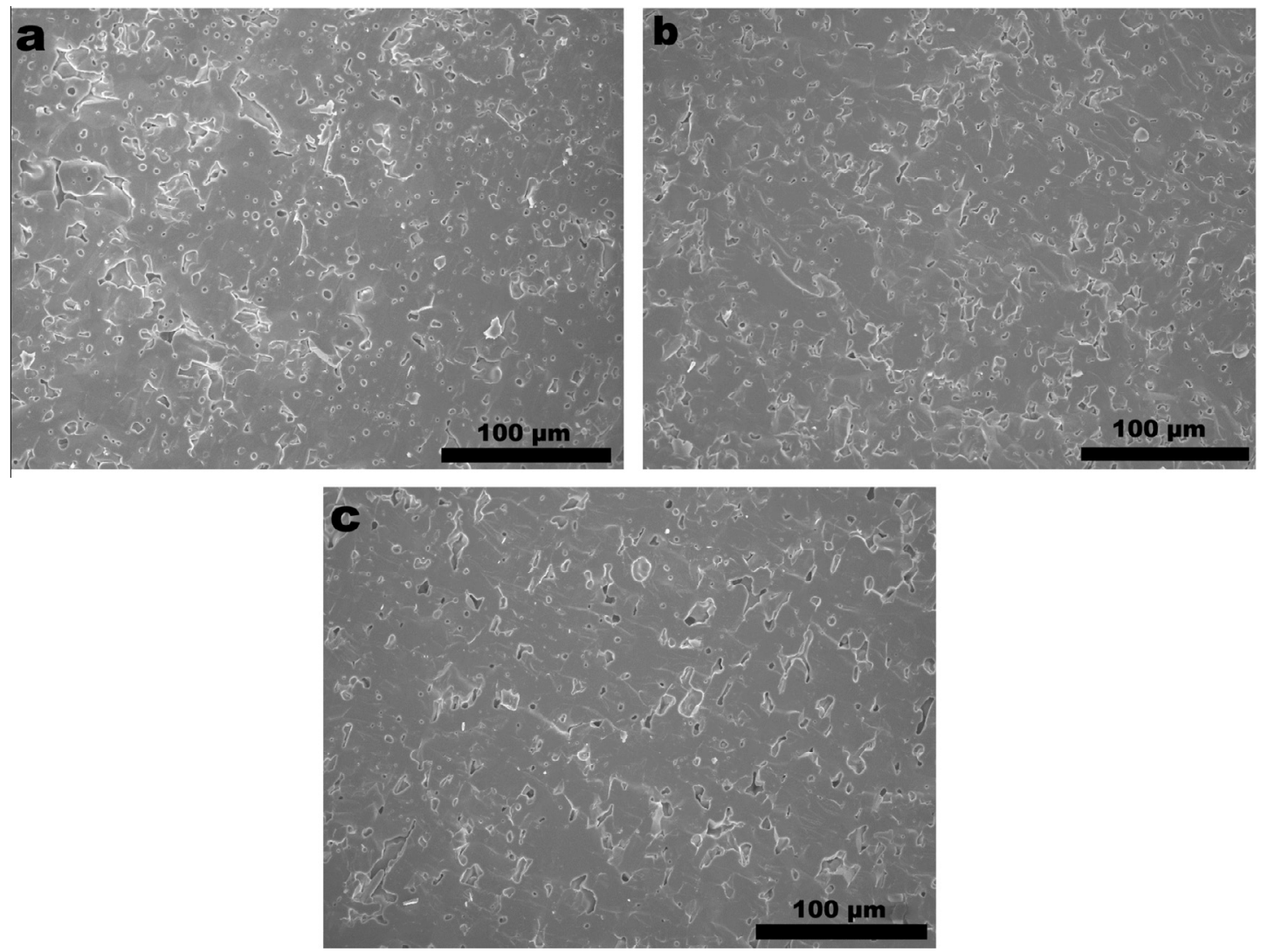

Fig. 2 - SEM images of the surfaces of (a) HA, (b) 0.5 wt.\% GNS/HA composite and (c) 1.0 wt.\% GNS/HA composite.

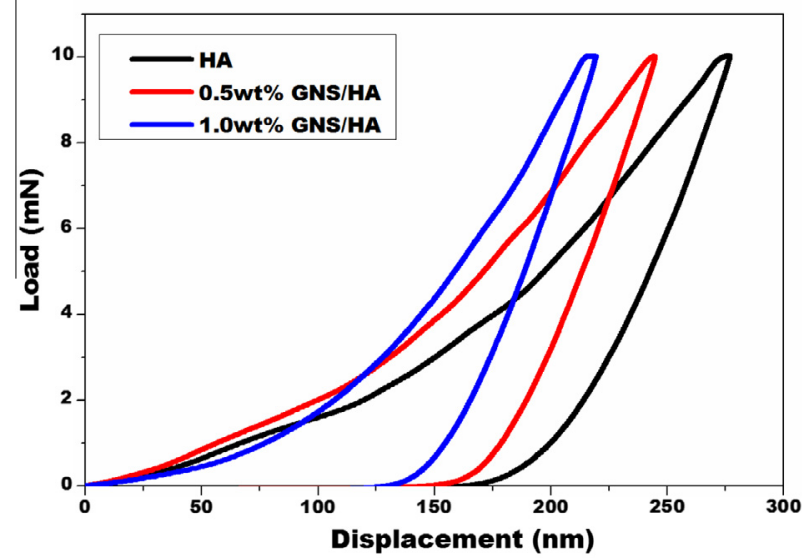

Fig. 3 - Representative load vs. displacement curves for HA, 0.5 wt.\% GNS/HA and 1.0 wt.\% GNS/HA composites. structure, and 2D peak is associated with few-layered graphene structure $[21,22]$. The presence of $G$ and $2 D$ peaks in the GNS/HA composites confirms the survival of GNSs even after undergoing harsh processing conditions. Shifting of D, G and $2 \mathrm{D}$ peaks to higher frequency for the GNS/HA composite is also observed as compared to the as-received GNSs, especially $\mathrm{G}$ peak shows a $\sim 15 \mathrm{~cm}^{-1}$ blue-shift. The compressive stress applied on GNSs during SPS might be the underlying reason for the blue-shift [22]. Intensity ratio between $D$ peak and $G$ peak $\left(I_{D} / I_{G}\right)$ is often used to quantify the degree of defects in graphene. As shown in Fig. $1 b$, the $I_{D} / I_{G}$ values of 0.5 wt. $\%$ GNS/HA and 1.0 wt. $\%$ GNS/HA composites are higher than that of as-received GNSs, illustrating that SPS process introduce structural defects into GNSs. Previous studies also reported that the intensity ratio of $I_{2 D} / I_{G}$ decreased with increasing number of graphene layers [21,22]. However, it is evident from Fig. 1b that 2D peak for $1.0 \mathrm{wt} \%$ GNS/HA composite is much narrower and sharper than that of as-received GNSs. This strongly implies that thinning of GNS agglomerates into few-layer graphene occurs during both processes of the powder mixing and SPS processing. As mentioned

\section{Table 1 - Mechanical properties of HA and GNS/HA composites.}

\begin{tabular}{llll} 
Samples & $E_{I T}(\mathrm{GPa})$ & $\mathrm{H}_{\mathrm{V}}(\mathrm{GPa})$ & $\mathrm{K}_{\mathrm{IC}}\left(\mathrm{MPa} \mathrm{m}^{0.5}\right)$ \\
\hline HA & $98 \pm 12.11$ & $5.51 \pm 0.67$ & $0.58 \pm 0.12$ \\
0.5 wt.\% GNS/HA composite & $124 \pm 6.99$ & $6.17 \pm 0.41$ & $0.80 \pm 0.01$ \\
1.0 wt.\% GNS/HA composite & $141 \pm 8.50$ & $7.22 \pm 0.17$ & $1.06 \pm 0.03$ \\
\hline
\end{tabular}



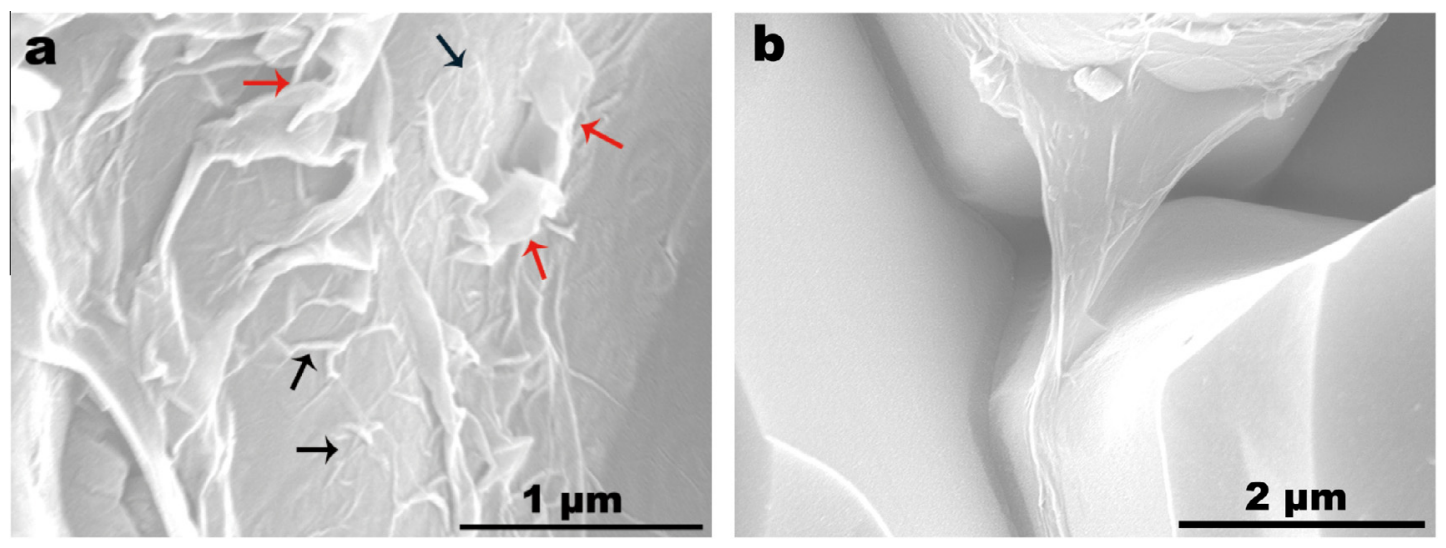

Fig. 4 - SEM images showing (a) wrinkled surface texture of a GNS wrapped around the HA grain (wrinkles are pointed out by black arrows and ridges are pointed out by red arrows), (b) grain bridging by GNS on the fracture surface of 1.0 wt.\% GNS/HA composite. (For interpretation of the references to colour in this figure legend, the reader is referred to the web version of this article.)
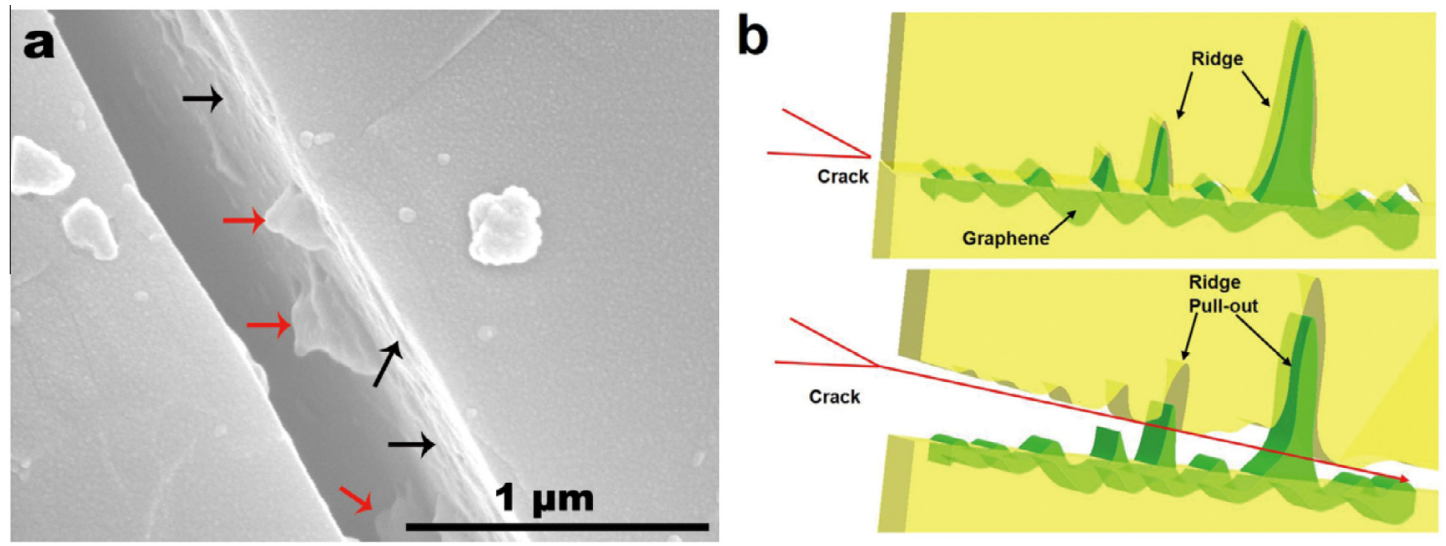

Fig. 5 - (a) SEM image showing Vickers indentation induced radial crack of 0.5 wt.\% GNS/HA composite, in which wrinkled texture (pointed out by black arrows) and GNS pull-out (pointed out by red arrows) are visible on the crack surface, (b) Schematic illustration of GNS pull-out in the GNS/HA composite. (For interpretation of the references to colour in this figure legend, the reader is referred to the web version of this article.)
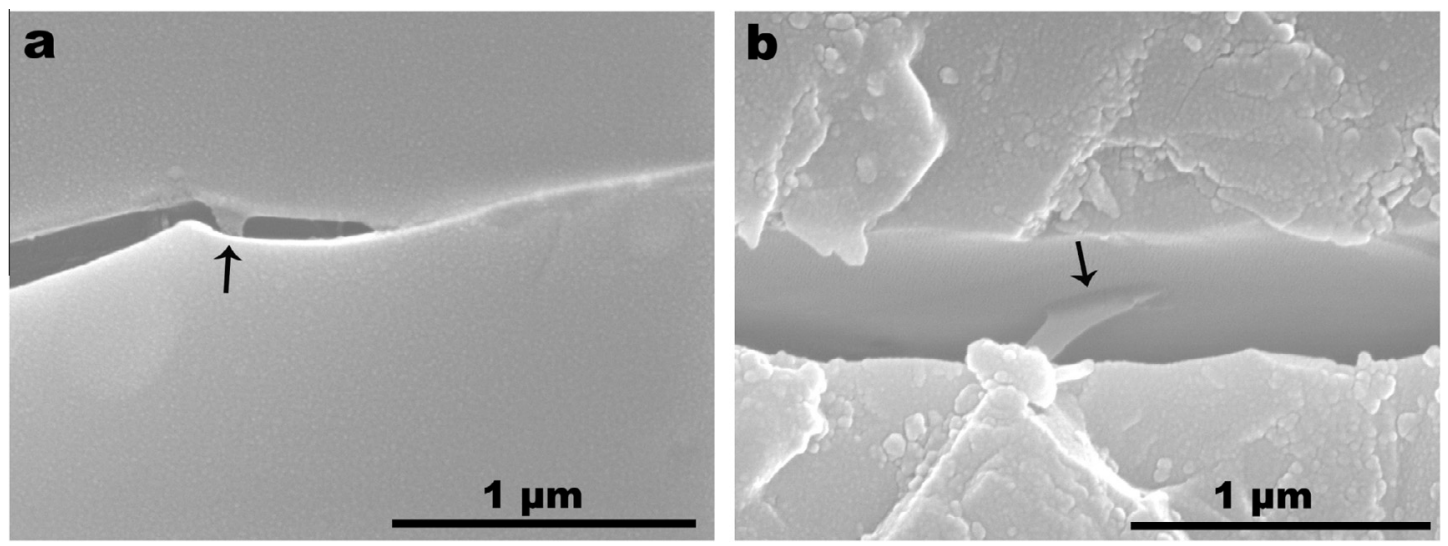

Fig. 6 - SEM images of (a) inhibition of the Vickers-indentation induced crack by a GNS, and (b) crack bridging by a GNS.

early, SDBS was used to disperse as-received GNS in the process of powder mixing. Hence, hydrophilic end of surfactant was connected with the water and the hydrophobic end of surfactant was attached with GNSs, thus it could disperse these agglomerated GNSs. Additionally, the localized spark discharge, high temperature and high applied pressure during 
SPS might also influence the structure of GNSs. Raman study of GPL/silicon nitride composites showed the similar trace of thinning of multilayered GPLs into few-layered ones [11].

Fig. 1c shows the XRD patterns of sintered samples containing different amounts of GNSs. It is clearly seen that the major peaks in the diffraction patterns are from HA (JCPDS PDF No. 9-432). The highest peak of $\alpha$-tri calcium phosphate $\left(\alpha\right.$-TCP) (JCPDS PDF No. 29-359, 20 $=30.71^{\circ}$ ) is absent in each samples, the second highest peak of $\alpha$-TCP $\left(2 \theta=22.89^{\circ}\right)$ overlaps with one peak of HA $\left(2 \theta=22 \cdot 90^{\circ}\right)$. However, it is noted that the third highest peak of $\alpha$-TCP $\left(2 \theta=34.21^{\circ}\right)$ is detected in the sintered HA and 1.0 wt.\% GNS/HA composites. On the other hand, the three highest peaks of $\beta$-TCP (highest peak: $2 \theta=30.9^{\circ}$, second highest peak: $2 \theta=34.37^{\circ}$ and third highest peak: $2 \theta=27.77^{\circ}$, JCPDS PDF No. 9-169) are all absent in each sample. XRD analysis sufficiently illustrate that few dissociation of HA into $\alpha$-TCP is occurred due to the rapid processing conditions and low sintering temperature $\left(1150^{\circ} \mathrm{C}\right)$ during SPS. Also, the surface morphologies of the sintered samples are shown in Fig. 2, it is found that the added GNSs seem to increase the porosity of the composite. Though an increase in porosity of a GNS/HA composite might more or less deteriorate its mechanical properties, these nano-scaled pores contribute to osteoblast ingrowth the composite.

Representative load-displacement curve of each sintered sample obtained through nanoindentation can be found in Fig. 3. Table 1 summarizes the measured elastic modulus, Vickers hardness and fracture toughness for each sample. It is clearly seen that elastic modulus and Vickers hardness for 1.0 wt.\% GNS/HA composite show improvements of $\sim 40 \%$ and $\sim 30 \%$, respectively, as compared to pure HA. Moreover, fracture toughness of a GNS/HA composite increases by up to $\sim 80 \%$ (from $\sim 0.58$ to $\sim 1.06 \mathrm{MPa} \mathrm{m}^{0.5}$ ) at $1.0 \%$ weight fraction. These results strongly indicate that added GNSs exhibits significant toughening effects on HA even at very low concentration.

To develop a comprehensive understanding of the contribution of the added GNSs to the improved mechanical properties, fractographic characteristics of GNS/HA composites were analyzed using SEM. As depicted in Fig. 4a, wrapping of a GNS around HA grain is clearly visible. The high specific surface area of GNSs is capable of forming an increased contact area with matrix. As a result, the bonding strength between GNS and HA grain could be significantly enhanced, and more energy would be required to make the nanofiller pull-out from HA matrix. Rough and wrinkled surface texture of the GNSs (as pointed out by black arrows in Fig. 4a) also plays an important role in enhancing mechanical interlocking, leading to an increased load-transfer efficiency between HA matrix and GNSs [23]. Fig. 4a shows ridges (as pointed out by red arrows) with a height of $\sim 300-400 \mathrm{~nm}$ protruding out of the wrinkled texture surface of the GNS. More interestingly, Fig. $4 \mathrm{~b}$ shows grain bridging by GNS on the fracture surface. It is clearly seen that the two ends of the GNS are well bonded to the adjacent HA grains with GNS plane nearly parallel to the fracture surface. For ceramics, it is well known that the toughness of
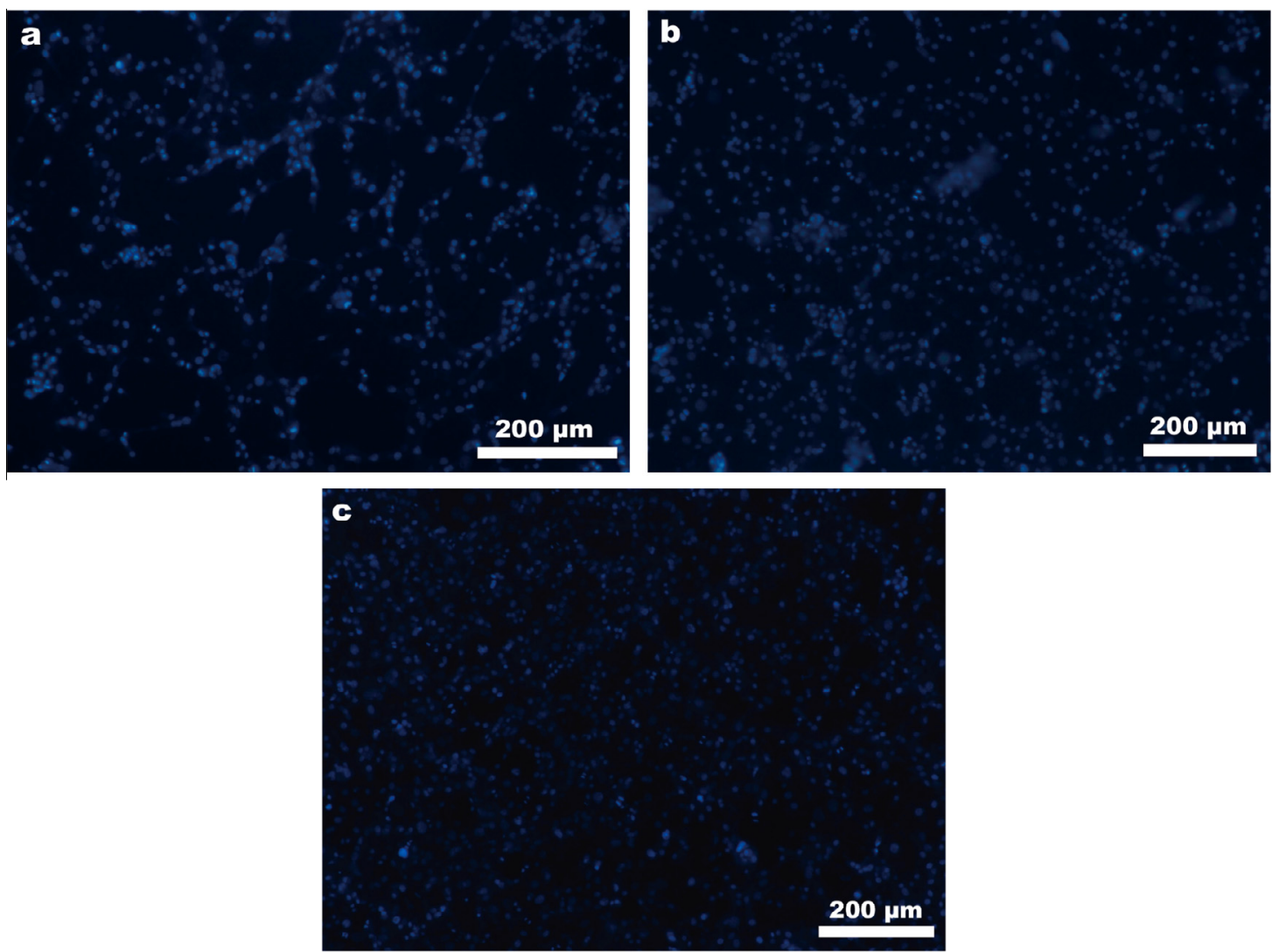

Fig. 7 - Fluorescent images of adherent osteoblasts on (a) HA, (b) 0.5 wt.\% GNS/HA composite and (c) 1.0 wt.\% GNS/HA composite for $6 \mathrm{~h}$ incubation. 
grain boundaries is lower than the grains. Thus, as a novel grain-boundary toughening mechanism, it is hypothesized that the grain bridging by GNS has a fundamental role in inhibiting crack propagation along grain boundary.

Further, typical characteristics of radial cracks induced by Vickers indentation were also observed using SEM. As clearly seen in Fig. 5a, some sheets, with a height of $\sim 300 \mathrm{~nm}$, protrude out of the plane of the crack surface (denoted by red arrows in Fig. 5a). Also, the wrinkled texture surface is faintly visible on the plane of the crack surface (denoted by black arrows in Fig. 5a). It is interesting to point out that the morphology and the height of these sheets protruding out of the plane of the crack surface are very similar to those of the ridges on the wrinkled texture surface of GNSs (Fig. 4a). When a crack propagates through the HA matrix and reaches a GNS across its path, the ridges on the GNS surface might be first to experience pull-out resulting in energy dissipation, due to binding and friction. Therefore, it is envisaged that GNS pull-out usually occurs from the interface between "GNS ridge" and matrix grain, as schematically illustrated in Fig. 5b. It is interesting to find that the Vickers indentation crack not only deflects its propagation direction due to the existence of GNS, but also the crack sometimes can be arrested by a GNS locating at the end of crack propagation path, as indicated in
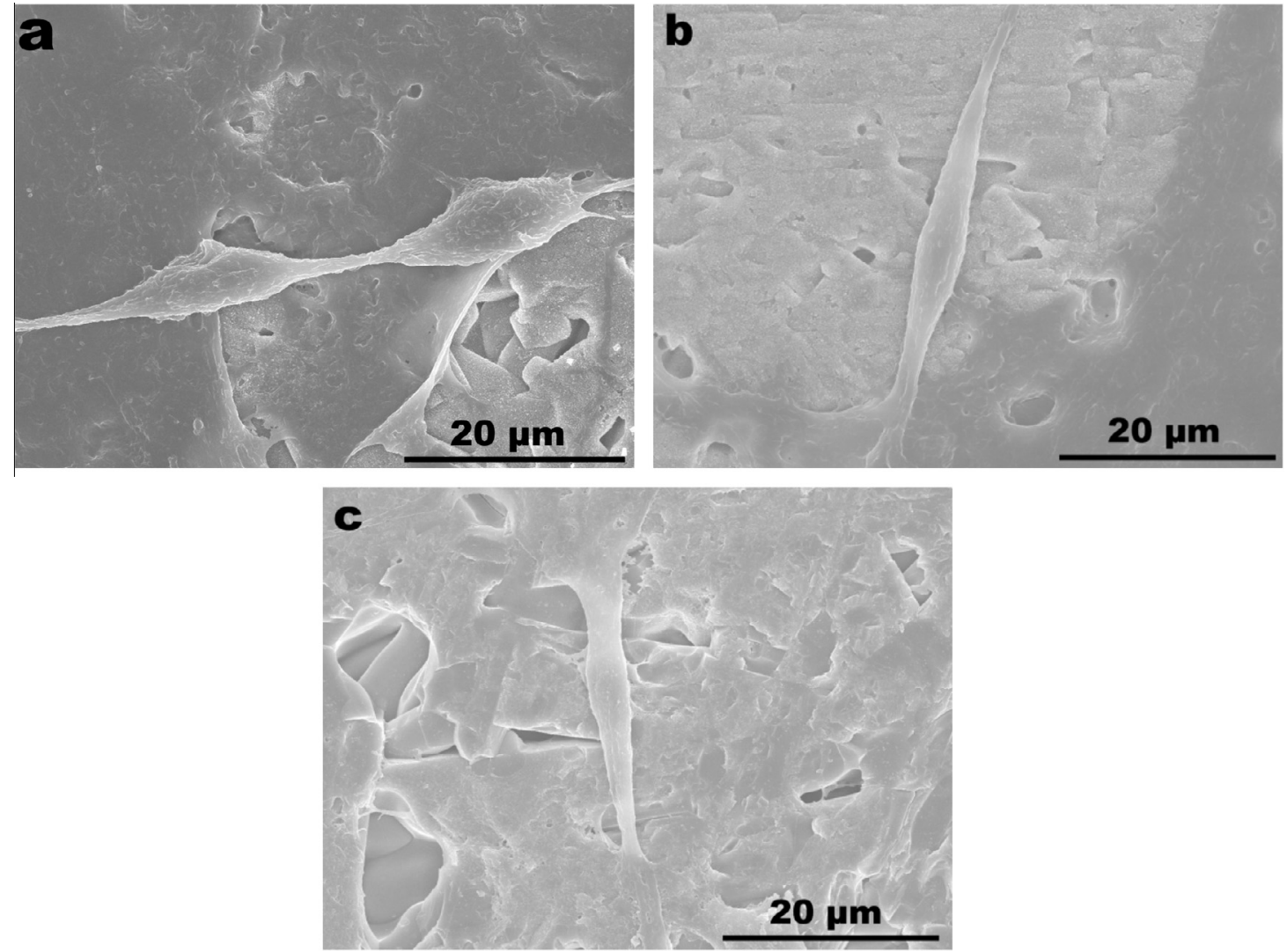

Fig. 8 - SEM images of the adherent osteoblasts on (a) HA, (b) 0.5 wt.\% GNS/HA composite and (c) 1.0 wt.\% GNS/HA composite.
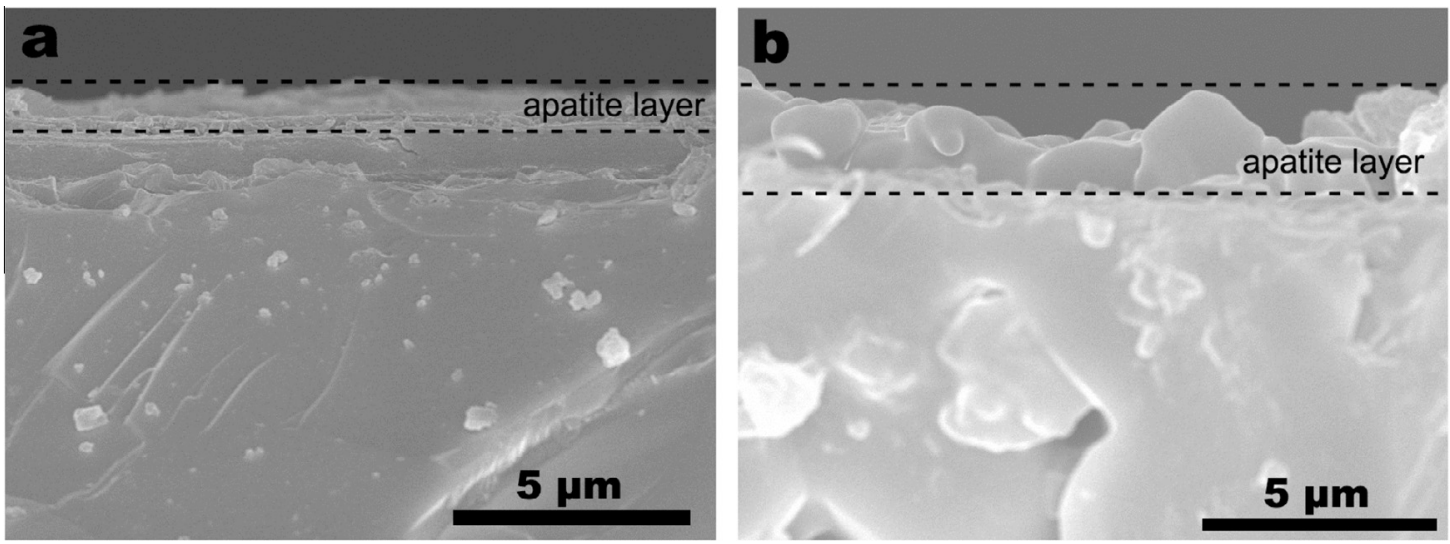

Fig. 9 - SEM images of the cross-section of (a) HA, (b) 1.0 wt.\% GNS/HA composite immersed into SBF for 7 days. 

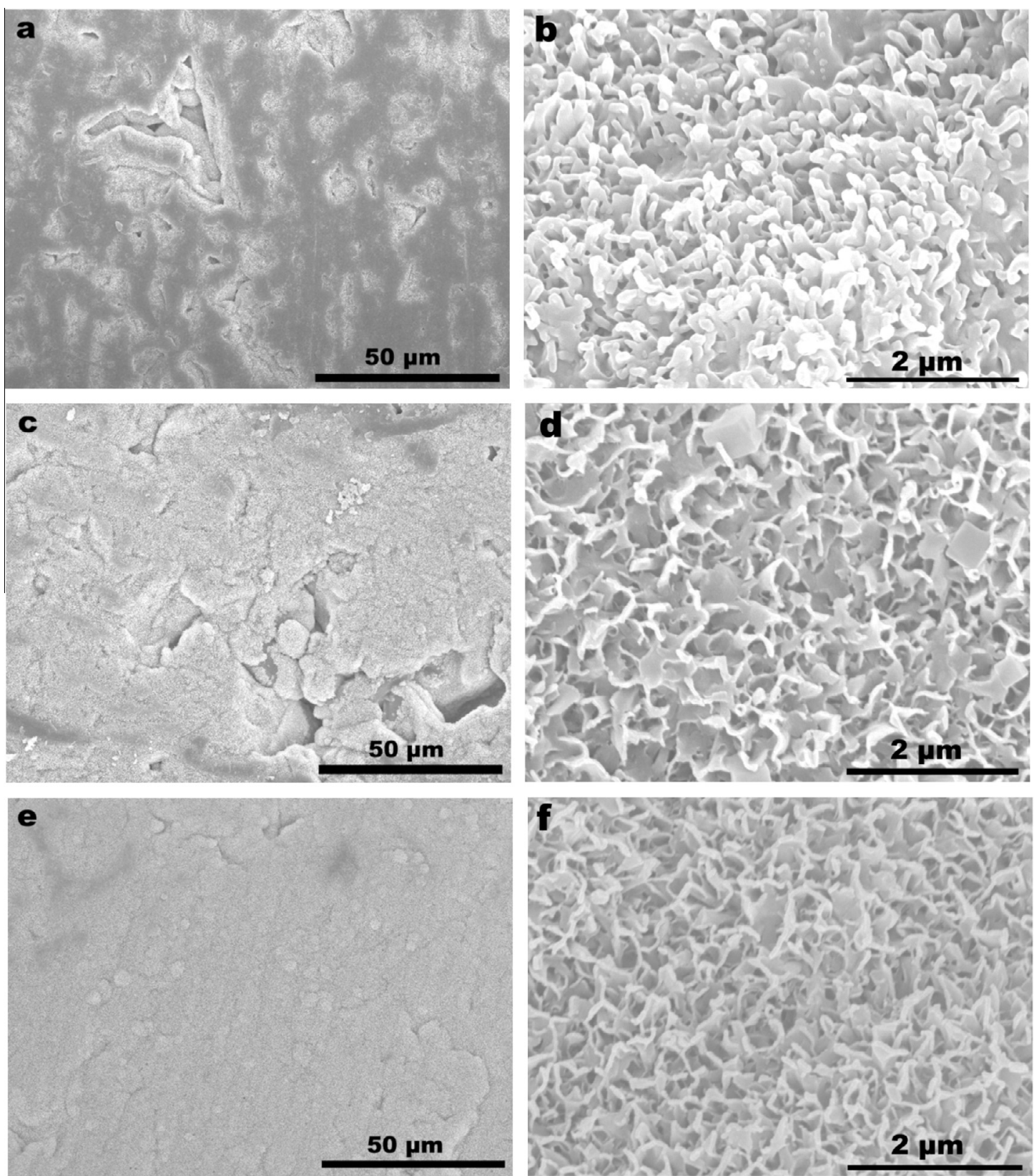

Fig. 10 - Low- and high-magnification SEM images of apatite formation on HA (a and b), 0.5 wt.\% GNS/HA composite (c and d) and 1.0 wt.\% GNS/HA composite (e and $f$ ) immersed in SBF for 7 days.

Fig. 6a. Crack bridging is a very frequently observed toughening mechanism in the GNS/HA composites. As seen in Fig. $6 \mathrm{a}$ and $b$, the bridging GNS retains its typical sheet-like structure and nearly parallels to the fracture surface. On the basis of above observations and analysis, we can infer that crack bridging and grain bridging by GNS, crack deflection and GNS pull-out are responsible for the improved fracture toughness of the GNS/HA composites.

\subsection{In vitro biocompatibility}

Adhesion of osteoblasts on the implants surface is an important prerequisite for its proliferation and formation of mineral deposits [17]. Also, it plays a crucial role in osseointegration to determine the life-time of the implants [13]. Fig. 7 shows that the amount of adherent osteoblasts increases with increasing GNS content. However, Lahiri et al. found that the survivability of osteoblasts deteriorates up to $86 \%$ on the surface of an ultrahigh molecular weight polyethylene composite reinforced by $1.0 \mathrm{wt} \%$ GNSs [24]. The effect of the content of the added GNSs in the GNS/HA composite system on the osteoblast viability will be focused in our future work. Osteoblasts uniformly cover the surface of the GNS/HA composites as a confluent layer as compared to the separate islands formed on the pure HA surface. Morphologies of the adherent osteoblasts on pure HA and GNS/HA composites are shown in Fig. 8. It is clearly seen that osteoblasts on the surface of each sample are elongated and flat with a good 
spread, strongly implying that the added GNSs have little negative effect on the osteoblast spreading on the HA sample derived from its excellent biocompatibility.

The difference in the number of adherent osteoblasts illustrate that GNSs impart the composite an optimal surface for osteoblast adhesion. Webster et al. [25] found an increased number of adherent osteobalsts when carbon nanofibres were added to polycarbonate urethane. It is likely due to an increase in material defects and electron delocalization on the nanostructured surface. Ribeiro et al. [26] reported that nano-roughness might enhance the osteoblast adhesion on the surface of bioceramics. Additionally, nanoscale topography of HA surface influences significantly the activity of osteoblast such as adhesion and proliferation, especially wide nanosheets $(>100 \mathrm{~nm})$ are favorable for osteoblast adhesion [27]. As discussed in Section 3.1, SPS process introduces structural defects into GNS (Fig. 1b). As a result, when GNSs are incorporated into HA matrix, the surface of the composite is believed to not only possess more defects, but also cause a change in nanoscale topography due to the presence of obvious wrinkled texture surface of GNSs (Fig. 4a). Therefore, it is hypothesized that the added GNSs could be detected by osteoblasts as extra suitable locations to adhere, and subsequently leading to the improved osteoblast adhesion on the GNS/HA composites.

Cross-sections of samples immersed into SBF for 7 days are depicted in Fig. 9, apatite layer on 1.0 wt.\% GNS/HA composite is obviously thicker than that on HA. Fig. 10 shows the representative surface morphologies of HA and the GNS/HA composites mineralized in SBF at $37^{\circ} \mathrm{C}$ for 7 days. It is clearly observed that the mineralization area on pure HA mainly localizes near and/or inside the pores, while mineral deposit ultimately pervades the whole surface of a GNS/HA composite. By comparison with Fig. 10b, $d$ and f, the morphology of mineralization product varies dramatically with incorporation of GNSs into HA matrix. Short-needle-shaped apatite

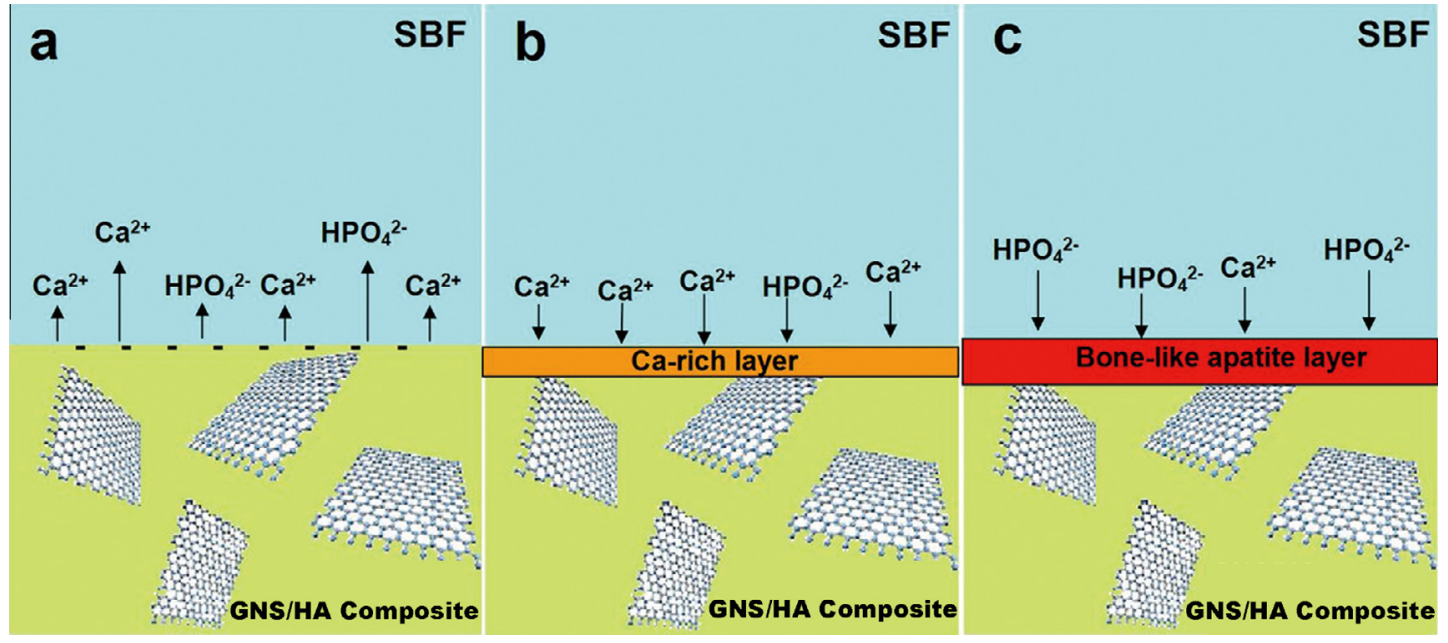

Fig. 11 - Schematic illustration of the mineralization process for a GNS/HA composite immersed in SBF. (a) Dissolution controlled stage, in which calcium ions dissolution is predominant, leading to negatively charged surface. (b) Precipitation controlled stage, in which negatively charged surface together with more nucleation sites attract calcium ions from SBF to form the Ca-rich layer. (c) Formation of bone-like apatite, in which Ca-rich layer attracts phosphate ions and form bone-like apatite.
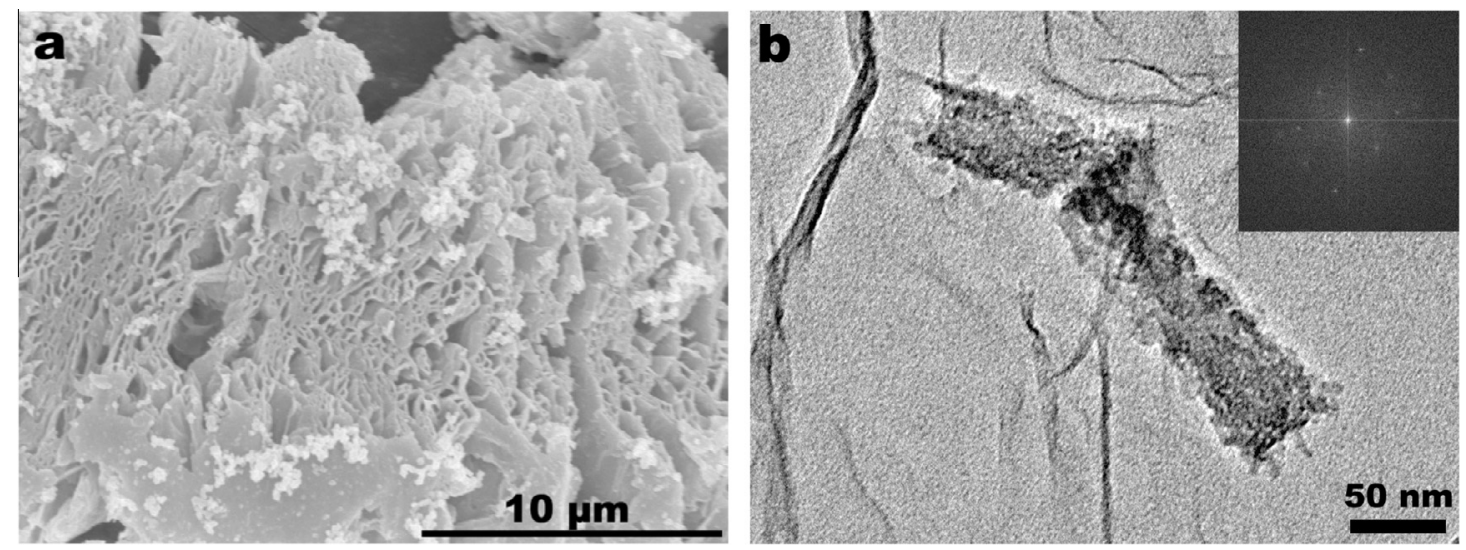

Fig. 12 - (a) SEM and (b) TEM images showing apatite formation on the surface of GNS immersed into SBF for 7 days. The inset is the selected area diffraction (SAD) pattern of the apatite. 
are formed on pure HA sample whereas curled-sheet-shaped apatite forms on GNS/HA composites.

These findings sufficiently indicate that incorporation of GNSs into HA matrix facilitates the formation of bone-like apatite on the GNS/HA composites. As depicted in Fig. 11, the possible mineralization process is proposed as follows. (1) Dissolution controlled stage. Zhang [28] analyzed the dissolution behavior of the HA coatings immersed in SBF and concluded that the dissolution of phosphate ions is dominated by crystallinity, while the dissolution of calcium ions is controlled by grain refinement. It is evident from XRD results (Fig. 1c) that the added GNSs have little effect on the crystallinity of the sintered composites, implying dissolution of the phosphate should be compatible for each sample in the present research. On the other hand, dissolution of calcium would be accelerated on the surface of a GNS/HA composite due to inhibition of HA grain growth by the additive GNSs on the grain boundaries $[9,10]$. Moreover, smaller grain size generally results in more specific area of the sample, which in turn benefits the interaction with SBF and ultimately increases dissolution of calcium ions. Hence, a GNS/HA composite immersed into SBF is expected to have higher negative charge than pure HA. Also, it should be noted that some GNSs are exposed in SBF at this dissolution-controlled stage. (2) Precipitation controlled stage. This stage is governed by the following factors such as the rich level of calcium ion in SBF, negatively charged surface and nucleation sites on the surface of a sample. For pure HA, the more HA dissolved, the more the nucleation sites created [28]. Moreover, it is believed that the defects of the exposed GNSs in contact with SBF act as nucleation sites, which is evidenced by the ability of apatite formation on the surfaces of the as-received GNSs. As depicted in Fig. 12a, lots of particles are observed on the surface of neat GNSs immersed in SBF for 7 days. TEM analysis (Fig. 12b) and EDS measurement on these particles demonstrates that the $\mathrm{Ca} / \mathrm{P}$ ratio is $\sim 1.50$, which is lower than that of pure $\mathrm{HA}(\mathrm{Ca} /$ $\mathrm{P}=1.67)$, but close to that of carbonated apatite $(\mathrm{Ca} / \mathrm{P}=1.63)$ [29], strongly indicating that GNSs possess an ability of apatite formation. As a result, higher concentration of calcium ion in SBF, higher negative charge together with more available nucleation sites permit a GNS/HA composite to form Ca-rich layer almost pervading the whole sample surface (Fig. 10c and e), while Ca-rich segments occur on the surface of pure HA sample (Fig. 10a). (3) Formation of bone-like apatite. Ca-rich layer attracts phosphate ions in SBF to form bone-like apatite cluster.

The difference in the morphological selection of mineralization product might ascribe to the precipitation behavior derived from the GNSs, which will be explored in our future investigation.

\section{Conclusions}

A free-standing GNS/HA composite was consolidated using SPS. Raman spectra evinced that GNSs retain their original structure even after experiencing harsh processing conditions. Compared to monolithic HA, the $1.0 \mathrm{wt} . \%$ GNS/HA composite displays $\sim 80 \%$ improvement in fracture toughness. This can be attributed to a combination of toughening mech- anisms including crack bridging and crack deflection, GNS pull-out and grain bridging by GNS. Grain bridging by GNS is the most effective mechanism to improve grain-boundary toughness. Results of osteoblast adhesion and apatite mineralization in SBF strongly illustrate that GNS/HA composite exhibits improved biocompatibility in vitro as compared pure HA. It is hypothesized GNSs not only provide more preferable locations for osteoblast adhesion, but also create more nucleation sites facilitating apatite mineralization.

\section{Acknowledgements}

Y.C. acknowledges financial supports from the National Natural Science Foundation of China (Grant No. 51275326), Natural Science Foundation of Jiangsu Province of China (Grant No. SBK2010212) and Opening fund of The State Key Laboratory of Nonlinear Mechanics. T.H.Z. is grateful for the financial supports from National Natural Science Foundation of China (Grant Nos. 11025212 and 11272318). Also, the authors would like to thank Dr. Meng Liu for her kindly help of biocompatibility tests.

\section{R E F E R E N C E S}

[1] Lee C, Wei XD, Kysar JW, Hone J. Measurement of the elastic properties and intrinsic strength of monolayer graphene. Science 2008;321(5887):385-8.

[2] Frank IW, Tanenbaum DM, Van der Zande AM, McEuen PL. Mechanical properties of suspended graphene sheets. J Vac Sci Technol B 2007;25(6):2558-61.

[3] Steurer P, Wissert R, Thomann R, Muelhaupt R. Functionalized graphenes and thermoplastic nanocomposites based upon expanded graphite oxide. Macromol Rapid Commun 2009;30(4-5):316-27.

[4] Nieto A, Lahiri D, Agarwal A. Synthesis and properties of bulk graphene nanoplatelets consolidated by spark plasma sintering. Carbon 2012;50(11):4068-77.

[5] Rafiee MA, Rafiee J, Srivastava I, Wang Z, Song HH, Yu ZZ, et al. Fracture and fatigue in graphene nanocomposites. Small 2010;6(2):179-83.

[6] Rafiee MA, Rafiee J, Wang Z, Song HH, Yu ZZ, Koratkar N. Enhanced mechanical properties of nanocomposites at low graphene content. ACS Nano 2009;3(12):3884-90.

[7] Bartolucci SF, Paras J, Rafiee MA, Rafiee J, Lee S, Kapoor D, et al. Graphene-aluminum nanocomposites. Mater Sci Eng A 2011;528(27):7933-7.

[8] Wang JY, Li ZQ, Fan GL, Pan HH, Chen ZX, Zhang D. Reinforcement with graphene nanosheets in aluminum matrix composites. Scr Mater 2012;66(8):594-7.

[9] Kun P, Tapasztó O, Wéber F, Balázsi C. Determination of structural and mechanical properties of multilayer graphene added silicon nitride-based composites. Ceram Int 2012;38(1):211-6.

[10] Kvetková L, Duszová A, Hvizdoš P, Dusza J, Kun P, Balázsi C. Fracture toughness and toughening mechanisms in graphene platelet reinforced $\mathrm{Si}_{3} \mathrm{~N}_{4}$ composites. Scr Mater 2012;66(10):793-6.

[11] Walker LS, Marotto VR, Rafiee MA, Koratkar N, Corral EL. Toughening in graphene ceramic composites. ACS Nano 2011;5(4):3182-90.

[12] Yu LG, Khor KA, Li H, Cheang P. Effect of spark plasma sintering on the microstructure and in vitro behavior of 
plasma sprayed HA coatings. Biomaterials 2003;24(16):2695-705.

[13] Lahiri D, Ghosh S, Agarwal A. Carbon nanotube reinforced hydroxyapatite composite for orthopedic application: a review. Mater Sci Eng C 2012;32(7):1727-58.

[14] Lahiri D, Benaduce AP, Rouzaud F, Solomon J, Keshri AK, Kos $\mathrm{L}$, et al. Wear behavior and in vitro cytotoxicity of wear debris generated from hydroxyapatite-carbon nanotube composite coating. J Biomed Mater Res A 2011;96A(1):1-12.

[15] Matsuoka M, Akasaka T, Totsuka Y, Watari F. Strong adhesion of saos-2 cells to multi-walled carbon nanotubes. Mater Sci Eng B 2010;173(1-3):182-6.

[16] Park S, Ruoff RS. Chemical methods for the production of graphenes. Nat Nanotechnol 2009;4(4):217-24.

[17] Kalbacova M, Broz A, Kong J, Kalbac M. Graphene substrates promote adherence of human osteoblasts and mesenchymal stromal cells. Carbon 2010;48(15):4323-9.

[18] Hu WB, Peng C, Luo WJ, Lv M, Li XM, Li D, et al. Graphenebased antibacterial paper. ACS Nano 2010;4(7):4317-23.

[19] Liu HY, Xi PX, Xie GQ, Shi YJ, Hou FP, Huang L, et al. Simultaneous reduction and surface functionalization of graphene oxide for hydroxyapatite mineralization. J Phys Chem C 2012;116(5):3334-41.

[20] Kokubo T, Kushitani H, Sakka S, Kitsugi T, Yamamuro T. Solutions able to reproduce in vivo surface-structure changes in bioactive glass-ceramic a-w3. J Biomed Mater Res 1990;24(6):721-34.

[21] Ferrari AC, Meyer JC, Scardaci V, Casiraghi C, Lazzeri M, Mauri F, et al. Raman spectrum of graphene and graphene layers. Phys Rev Lett 2006;97(18):187401.
[22] Ni ZH, Wang HM, Ma Y, Kasim J, Wu YH, Shen ZX. Tunable stress and controlled thickness modification in graphene by annealing. ACS Nano 2008;2(5):1033-9.

[23] Lahiri D, Khaleghi E, Bakshi SR, Li W, Olevsky EA, Agarwal A. Graphene-induced strengthening in spark plasma sintered tantalum carbide-nanotube composite. Scr Mater 2013;65(5):285-8.

[24] Lahiri D, Dua R, Zhang C, de Socarraz-Novoa I, Bhat A, Ramaswamy S, et al. Graphene nanoplatelet-induced strengthening of ultrahigh molecular weight polyethylene and biocompatibility in vitro. ACS Appl. Mater. Interfaces 2012;4(4):2234-41.

[25] Webster TJ, Waid MC, McKenzie JL, Price RL, Ejiofor JU. Nanobiotechnology: carbon nanofibres as improved neural and orthopaedic implants. Nanotechnology 2004;15(1):48-54.

[26] Ribeiro N, Sousa SR, Monteiro FJ. Influence of crystallite size of nanophased hydroxyapatite on fibronectin and osteonectin adsorption and on MC3T3-E1 osteoblast adhesion and morphology. J Colloid Interface Sci 2010;351(2):398-406.

[27] Okada S, Ito H, Nagai A, Komotori J, Imai H. Adhesion of osteoblast-like cells on nanostructured hydroxyapatite. Acta Biomater 2010;6(2):591-7.

[28] Zhang Q. Dissolution and mineralization behaviors of HA coatings. Biomaterials 2003;24(26):4741-8.

[29] Kim HM, Himeno T, Kokubo T, Nakamura T. Process and kinetics of bonelike apatite formation on sintered hydroxyapatite in a simulated body fluid. Biomaterials 2005;26(21):4366-73. 